\title{
Lower Serum Irisin Levels Are Associated with Increased Osteoporosis and Oxidative Stress in Postmenopausal
}

\author{
Ali Badr Roomi*1,2, Wassan Nori ${ }^{3}$, Roaa Mokram Hamed ${ }^{4}$
}

\begin{abstract}
Background: Irisin as an exercise-induced myokine was proposed to improve bone health. This study investigated the role of serum irisin (s-irisin) in patients with osteoporosis (OP) through correlating to most biological bone markers and oxidative stress.

Methods: A cross-sectional study recruited an eligible 175 postmenopausal women at Al-Hussien Teaching Hospital, Iraq. They were scanned by DEXA and stratified into two groups based on T-score; the first 95 patients as control group (GI) with $-1 \leq \mathrm{T}$-score and the second 80 patients as cases group (GII) with T-score $\leq$ -2.5. Demographic criteria were age, bone mineral density $\left(\mathrm{BMD}, \mathrm{g} / \mathrm{cm}^{2}\right)$ and T-score. Serum irisin, total serum calcium (s-calcium), serum inorganic phosphate (s-phosphate), serum alkaline phosphatase (s-ALP), serum $25[\mathrm{OH}]$ vitamin $\mathrm{D}$, the serum parathyroid hormone (s-PTH), serum Carboxy terminal collagen crosslinks (CTx), serum procollagen type I C-termidnal peptide (s-PICP), serum malondialdehyde (s-MDA) and serum superoxide dismutase (s-SOD) were collected from blood samples.

Results: Serum irisin were $31.84 \pm 2.65$ vs. $20.88 \pm 2.71 \mathrm{ng} / \mathrm{mL}$ for control and trial groups, respectively. Lower levels of $\mathrm{BMD}$, T-score, $25[\mathrm{OH}]$ vitamin $\mathrm{D}$, and s-irisin along with a higher serum levels of PTH, CTx, PICP, MDA and SOD were observed in patients with osteoporosis. All parameters were statistically meaningful upon correlation $(\mathrm{p}<0.0001)$, except age and $\mathrm{s}$-calcium $(\mathrm{p}=$ 0.0088 and $\mathrm{p}=0.187$, respectively).

Conclusions: The results showed that, a significantly lower serum irisin levels among osteoporosis women, was intimately correlated to most bone turnover markers and it can be considered as encouraging results for clinical application in prediction and treatment of osteoporosis.
\end{abstract}

Keywords: Bone turnover markers, DEXA scan, Irisin, T-score, BMD, osteoporosis, post-menopause.

\section{Introduction}

Osteoporosis (OP) is characterized structurally by bone mineral density (BMD) T-score -2.5 or less. $\mathrm{OP}$ is associated with an aging population particularly for women during postmenopausal years. Menopause is a major etiological factor for $\mathrm{OP}$; a daunting period when females are most vulnerable to climate change and hormone degradation (1-3). BMD assessed with dualenergy X-ray absorptiometry (DXA) rigorously predicts substantial fractures, especially those involving the hip. Predictions strengthened when $\mathrm{BMD}$ is paired with additional diagnostic risk factors in predictive modules $(4,5)$.

Numerous risk prediction methods have been suggested, both radiological and biological modules, aiming for the detection of individuals at high risk of fracture that are mostly like to get the maximum benefit from treatment (6). Dynamic changes in bone metabolism especially turn-over markers may be best estimated by assessing 
different bone biological markers in the blood and urine, whereas changes in BMD evolve very slow and do not reflect the actual magnitude of bone loss. The latest studies have highlighted the possible use of bone turnover markers (BTMs) to classify patients with early bone loss, to make proper clinical choices, and to track therapy outcomes. Harmonization of bone turnover markers can promote bone turnover markers for the prediction of fracture risk (7).

None of the prediction modules cover all established risk factors each have their strengths and weaknesses in routine clinical practice $(7,8)$. Irisin is a novel endocrine myokine marker produced in an exercise that works by inducing browning of the adipose tissue. The latter is a positive indicator of femoral bone architecture and is directly related to thigh muscle. Interestingly; irisin increased osteoblast differentiation, and its levels were lower among women with a history of osteoporotic fractures. Irisin is also secreted by fatty tissue, in the experimental animals, highlighting its action in the muscle-fat-bone cross-talk (9-11). A growing body of evidence correlates s-irisin and osteoporotic fractures still the earlier study did not specify the irisin role in that relationship. The current study was designed to explore irisin correlation in OP as a possible predictor, our knowledge for its role among Iraqi women is scrutinized.

\section{Materials and Methods}

This study was a prospective cross-sectional study involving 175 postmenopausal Iraqi women PMIW at Al-Hussien Teaching Hospital in ThiQar City over one year time; from January 2020 to March 2020.

\section{Ethics approval}

Ethical approval was made by the local ethics committee together with verbal approvals by all participants. PMIW were those who experienced 12 months of amenorrhea consecutively and $40 \mathrm{mIU} / \mathrm{ml}$ of folliclestimulating hormone providing there is no medical reason. Exclusion criteria included rheumatic disease, or urinary, gout, drug abuse, alcohol use, smoking history. History of blood disorders, pathological fracture, diabetes hypertension, and irregular (renal, liver, or thyroid function test). We also removed volunteers with drugs and supplements that affect bone metabolism, vitamin $\mathrm{D}$, calcium supplements, bisphosphonates, and fluoride glucocorticoids.

\section{Experimental design}

The recruited volunteers who were eligible for inclusion criteria were 175 participants. They were scanned by the DEXA scan and further subdivided into two groups; the control group (95/175) referred to as G I, and the cases group (80/175) referred to as G II based on their DEXA scan. The Control group had $(-1 \leq \mathrm{T}$-score), Cases; OP group had (T-score $\leq-2.5)$. Demographic parameters for participants include age, from the radiological analysis, we estimated BMD $(\mathrm{g} / \mathrm{cm} 2)$ and T-score. From blood samples, we collected s-irisin, s-calcium, s-phosphate, sALP, serum $25[\mathrm{OH}]$ vitamin D, s-PTH, serum CTx (s-CTx), serum PICP (s-PICP), serum MDA, and serum SOD.

\section{BMD measurement}

Dual-energy X-ray absorptiometry [DEXA, Ltd., Korea/ Osteosys Co.] was used to calculate BMD and T-score at the lumbar spine (L2-L4). A procedure used for the DEXA test, the patient is placed in a supine position below the radiation source indicated by a red laser pointer, which steadily travels from the umbilical to the middle thigh known as the Ward triangle, over a test;

DEXA scans the femur neck, the commonest location for fractures. Height and body weight were also assessed when scanning for bone density was in progress.

T-score values estimated by DEXA scan are the standard for OP diagnosis. According to WHO criteria for the diagnosis of osteoporosis. Participants are sub- divided into three: regular (T-score value > 1.0 S.D), osteopenia (1.0 S.D T-score value > 2.5 S.D), and osteoporosis (T-score value 2.5 S.D). 


\section{Laboratory measurement}

Samples of blood were obtained in all volunteers between 8:30 and 12:00 a.m. They were collected, frozen for chemical processing in the enzyme-linked immunosorbent assay (ELISA) assay. Serum irisin, s-CTx, s-PICP, serum $25[\mathrm{OH}]$ vitamin $\mathrm{D}$, and s-PTH, were measured using the ELISA kit provided by Demeditec diagnostic testing GmbH Germany. S-calcium, s-phosphate, s-ALP calculated by (Biolabs), (France, man). The serum MDA amount was spectrophotometrically calculated using the Muslih et al. method in 2002 (12). Serum levels of SOD were assessed using the Superoxide Dismutase Microplate Assay Kit provided by Cohesion Biosciences USA. All samples were examined in one set.

\section{Statistical analysis}

The unpaired T-test was used to compare two mean \pm SDs for age, s-irisin, s-calcium, sphosphate, s-ALP, serum $25[\mathrm{OH}]$ vitamin $\mathrm{D}$, sPTH, s-CTx, s-PICP, serum MDA, and serum SOD all obtained from blood analysis. We calculated $\mathrm{BMD}$ and $\mathrm{T}$-score by radiological tests. For both calculations, the relevant P-value was $<0.05$. To clarify the correlation between irisin and other parameters we used Linear regression. Both statistical analyzes were carried out using Statistical Package Social Sciences version 24 (SPSS v.24).

\section{Results}

A total of 175 postmenopausal women volunteered to study. Their demographic criteria were summarized in Table 1, including, age, s-irisin, s-calcium, sphosphate, s-ALP, serum $25[\mathrm{OH}]$ vitamin D, the s-PTH, s-CTx, s-PICP, serum MDA, and serum SOD all yielded via blood analysis; the radiological exam estimated $\mathrm{BMD}(\mathrm{g} / \mathrm{cm} 2)$ and T-score.

The mean for the age was $58.41 \pm 3.51$ versus $59.73 \pm 3.44$ for GI and GII respectively, s-irisin $(\mathrm{ng} / \mathrm{mL})$ had a mean of $31.84 \pm 2.65$ versus $20.88 \pm 2.71$ for GI and GII respectively. All the variables taken by the study were significant upon comparison among the 2 groups as the p-value was < 0.0001 except for age and s-calcium with a pvalue of 0.0088 and 0.187 respectively.

Table 2 demonstrated the correlations of sirisin versus all parameters under study. A significant positive correlation was reached between irisin versus the BMD, s-phosphate, serum $25[\mathrm{OH}]$ vitamin $\mathrm{D}$, and s-PICP in the GI and GII groups. While, a significant negative correlation was proved between sirisin with T-score, s-ALP, s-PTH, s-CTx, serum MDA, and serum SOD in the G1 and GII groups. Irisin showed a non-significant negative correlation with s-calcium.

Table 1. Basic criteria for study volunteer-involving healthy controls and cases.

\begin{tabular}{|c|c|c|c|}
\hline Variable & GI: Control Group (n=95) & GII: Study Group (n= 80) & p-value \\
\hline Age & $58.41 \pm 3.51$ & $59.73 \pm 3.44$ & 0.0088 \\
\hline s-irisin (ng/mL) & $31.84 \pm 2.65$ & $20.88 \pm 2.71$ & $<0.0001^{*}$ \\
\hline $\operatorname{BMD}\left(\mathrm{g} / \mathrm{cm}^{2}\right)$ & $1.16 \pm 0.14$ & $0.55 \pm 0.14$ & $<0.0001 *$ \\
\hline T-score & $-0.91 \pm 0.13$ & $-4.32 \pm 0.79$ & $<0.0001 *$ \\
\hline s-calcium (mmol/L) & $2.24 \pm 0.16$ & $2.28 \pm 0.22$ & $0.186^{\mathrm{ns}}$ \\
\hline s-phosphate (mmol/L) & $1.86 \pm 0.13$ & $1.59 \pm 0.19$ & $<0.0001 *$ \\
\hline s-ALP (IU/dL) & $78.93 \pm 4.78$ & $85.83 \pm 4.79$ & $<0.0001^{*}$ \\
\hline $25[\mathrm{OH}]$ vitamin $\mathrm{D}(\mathrm{ng} / \mathrm{mL})$ & $26.56 \pm 2.42$ & $9.64 \pm 2.02$ & $<0.0001 *$ \\
\hline s-PTH (ng/ml) & $30.50 \pm 3.23$ & $38.17 \pm 3.66$ & $<0.0001^{*}$ \\
\hline s-CTx (ng/mL) & $0.39 \pm 0.08$ & $2.11 \pm 0.58$ & $<0.0001 *$ \\
\hline s-PICP (ng/mL) & $18.54 \pm 0.36$ & $18.07 \pm 0.41$ & $<0.0001 *$ \\
\hline MDA (mmol/l) & $4.83 \pm 0.27$ & $10.01 \pm 1.02$ & $<0.0001 *$ \\
\hline SOD $(\mathbf{U} / \mathbf{m l})$ & $2.88 \pm 0.37$ & $4.52 \pm 0.61$ & $<0.0001^{*}$ \\
\hline
\end{tabular}

s-irisin, serum irisin; BMD, bone mineral density; s-calcium, serum total calcium; s-phosphate, serum inorganic phosphate: s-ALP, alkaline phosphatase; s-PTH, parathyroid hormone; s-CTx, Serum human C terminal telopeptides of types I collagen; s-PICP, Serum human carboxyterminal propeptide of type I procollagen; MDA, Malondialdehyde; SOD, Superoxide dismutase. ${ }^{\text {ns }}$ non-significant; $*$ p value $<0.001$. 
Table 3, illustrated person correlations between BMD versus all other variables. Correlations were significant except for s- calcium as $\mathrm{p}<0.158$, the coefficient of correlation was most significant for MDA, -0.870 .

Table 2. The correlations between s-irisin versus all other variables.

\begin{tabular}{lcc}
\hline Variable & $\mathrm{R}$ & P-value \\
\hline BMD $\left(\mathrm{g} / \mathrm{cm}^{2}\right)$ & 0.828 & $<0.0001^{*}$ \\
T-score & -0.575 & $<0.0001^{*}$ \\
s-calcium $(\mathrm{mmol} / \mathrm{L})$ & -0.032 & $0.652^{\mathrm{ns}}$ \\
s-phosphate $(\mathrm{mmol} / \mathrm{L})$ & 0.551 & $<0.0001^{*}$ \\
s-ALP $(\mathrm{IU} / \mathrm{dL})$ & -0.525 & $<0.0001^{*}$ \\
25 $[\mathrm{OH}] \mathrm{vitamin} \mathrm{D}(\mathrm{ng} / \mathrm{mL})$ & 0.877 & $<0.0001^{*}$ \\
s-PTH $(\mathrm{ng} / \mathrm{ml})$ & -0.636 & $<0.0001^{*}$ \\
s-CTx $(\mathrm{ng} / \mathrm{mL})$ & -0.809 & $<0.0001^{*}$ \\
s-PICP $(\mathrm{ng} / \mathrm{mL})$ & 0.403 & $<0.0001^{*}$ \\
MDA $(\mathrm{mmol} / \mathrm{l})$ & -0.847 & $<0.0001^{*}$ \\
SOD $(\mathrm{U} / \mathrm{ml})$ & -0.774 & $<0.0001^{*}$ \\
\hline non-significant $*$ p value $<0.001$ & &
\end{tabular}

Ns: non-significant; $* \mathrm{p}$ value $<0.001$.

Table 3. The correlations between BMD versus all other variables.

\begin{tabular}{lcc}
\hline Variable & $\mathbf{R}$ & p-value \\
\hline S-irisin (ng/mL) & 0.828 & $<0.0001^{*}$ \\
T-score & -0.612 & $<0.0001^{*}$ \\
s-calcium (mmol/L) & -0.100 & $0.158^{\mathrm{ns}}$ \\
S-phosphate (mmol/L) & 0.496 & $<0.0001^{*}$ \\
S-ALP (IU/dL) & -0.512 & $<0.0001^{*}$ \\
25 [OH] vitamin D (ng/mL) & 0.859 & $<0.0001^{*}$ \\
S-PTH (ng/ml) & -0.608 & $<0.0001^{*}$ \\
S-CTx (ng/mL) & -0.820 & $<0.0001^{*}$ \\
S-PICP (ng/mL) & 0.433 & $<0.0001^{*}$ \\
MDA (mmol/l) & -0.870 & $<0.0001^{*}$ \\
SOD (U/ml) & -0.771 & $<0.0001^{*}$ \\
\hline
\end{tabular}

Ns: non-significant; $*$ p value $<0.001$.

\section{Discussion}

Despite impressive advances in understanding how estrogen deficiency causes OP, the underlying pathogenic pathways are diverse and multifaceted. One of the most intriguing theories is the capacity of bone turnover markers to predict OP in the affected bone as the changes precede the radiological changes Harmonization of bone markers, can promote prediction of OP and fracture risk. The newly discovered irisin, an exercise-induced myokine, originating from fibronectin type III domain-containing protein 5 (FNDC5) and released into the serum as a soluble form (known as irisin) was discussed in an attempt to verify its role in $\operatorname{OP}(13,14)$.

The most recent guidelines, recommend the use of vitamin D with a daily dosage of 800 IU for postmenopausal women and men $>50$ years old as for higher intermittent weekly doses; they were not advised (15). According to the present study's results, BMD and T-score were significantly lower in comparison to control. These results were succinct in the Roomi et al. study, where BMD $\left(\mathrm{g} / \mathrm{cm}^{2}\right)$ and T-score were substantially lower in OP patients as compared to stable controls (16).

Correlations indicate meaningful relations for $\mathrm{s}$-CTx $(\mathrm{ng} / \mathrm{mL})$ and s-PICP $(\mathrm{ng} / \mathrm{mL})$ as p-value< 0.0001 , amongst cases versus controls. These are biological markers for bone turnover, elevated after menopause. Since bone loss velocity differs by marker value; laboratory medicine (IFCC) has suggested two markers as discriminated 
analyses for prediction; serum procollagen type I $\mathrm{N}$ propeptide (s-PINP) and s-CTx as markers for bone formation and bone resorption, respectively. Researchers explored the relationship between PINP and the likelihood of fracture. Risk ratio raises in s-PINP; 1.23 (95\% CI 1.09-1.39) for males and females paired uncorrected for BMD. A substantial correlation was also identified for s-CTx and fracture risk $\mathrm{GR}=1.18(95 \%$ CI 1.05-1.34) uncorrected for BMD. The correlation between s-CTx and fracture risk was marginally higher for the consequence of the hip fracture, 1.23 (95 percent CI 1.04-1.47) There is also a minor but important association between such markers and the fracture risk (17).

Low intake $\mathrm{Ca}$ results in lower serum levels correlates to low BMD and postmenopausal OP; sufficient bone mineralization is obtained by calcium and vitamin D enriched dietary consumption. Calcium increases the mechanical and structural bone qualities of PMW bones. These results became the foundation for promoting an improved dietary fiber intake that could help enhance its absorption via the intestines. These undigested fibers will be ferment by the intestinal bacteria that lower intestinal PTH and promote $\mathrm{Ca}$ absorption to emphasize its protective action $(18,19)$.

Based on the present results, PTH serum levels were meaningfully higher in cases versus healthy controls. PTH is prescribed for treating postmenopausal women with osteoporosis, male osteoporosis, and corticosteroid-induced osteoporosis, which induces substantially elevated BMD of the lumbar spine (20).

Chawla et al illustrated a negative correlation between PTH and BMD. Interestingly; upon histomorphometry of iliac crest biopsies; increased BMD shows a compromised bone with increased cortical and trabecular width and reduced cortical porosity consequently, microarchitecture changes on skeletal health and silent vertebral fractures were reported (21).

In the animal model experiment, the concentrations of bone Calcium and $\mathrm{p}$ in treated ovariectomized mice were significantly lower than those in the control group; in line with $\mathrm{El}$ Wakf et al, which reported that ovariectomized mice have impaired $\mathrm{Ca}$ balance ascribed to ovariectomy-induced OP (22). Kharode (23) indicated that menopause causes an increase in bone resorption; and a net loss of bone. The cessation of ovarian hormones and the decreased secretion of estrogen was the trigger (24).

In the present study, analyses have shown significant differences in irisin levels in cases vs. controls ( $\mathrm{p}<$ 0.0001). Table 2 showed a significant correlation with all variables. Irisin positively correlates with BMD, s-phosphate, 25 $[\mathrm{OH}]$ vitamin $\mathrm{D}$, s-CTx, negatively correlate to s-ALP, s-PTH, s-CTx, MDA, and SOD. Table 3 illustrates person correlations between BMD vs. $s$-irisin and all other variables. Correlations were significant except for s-calcium ( $\mathrm{p}<0.158)$.

Radiological screening for OP has its disadvantages, inadequate diet in the elderly affects bone density expressed as osteomalacia, and reduced bone mineralization can lower the overall bone matrix. On the other hand, bone biomarkers will increase throughout menopause each with a different OP-related rate, combined modules will display higher identification and improved clinical outcomes (25).

Irisin's, positive influence on bone is defined as enhancing bone quality by increasing the rate of mineral deposition, bone synthesis, periosteal diameter, and polar bending momentum (26). Irisin was recently reported to impair osteoclast activity and stimulates osteoblast differentiation in bone cell lines it regulates bone anabolism indirectly via browning of the WAT [white adipose tissue] (26). Moreover, irisin reduces body fat, prevents and treats obesity by increasing energy expenditure. Moreover, proinflammatory cytokines caused by accumulated fat (27) can be reduced by irisin especially induced by exercise ultimately so that reduces osteoclast activity and increases osteoblast differentiation (28).

Results in rats' models have declared that irisin is involved in the bone-muscle talk. Experiments in humans aimed to investigate the irisin effect on the skeleton, postmenopausal women showed a negative relation in irisin levels and vertebral fragility fractures. Conversely, its levels were directly correlated with bone strength and density, among athletes 
confirming the irisin protective role in bone health (29).

However, the irisin effect extends to include the central nervous system. Irisin attracted the attention for a possible application in neurodegenerative diseases, such as Alzheimer's and Parkinson's (30,31).

In summary, Irisin has favorable effects on bone health due to its promising relation to bone turn over markers and negative relation to other bone-oxidative stress markers. Irisin's effect on bone prosperity is mediated via multiple pathways affecting bone cells, increasing

\section{References}

1. Leslie WD, Morin SN. New Developments in Fracture Risk Assessment for Current Osteoporosis Reports. Curr osteoporos Rep. 2020;18(3):115-29.

2. Fuleihan GE, Chakhtoura M, Cauley JA, Chamoun N. Worldwide fracture prediction. J Clin Densitom. 2017;20(3):397-424.

3. Duque, G. (Ed.). Osteosarcopenia: Bone, Muscle and Fat Interactions. Springer. 2019.

4. Shepstone L, Lenaghan E, Cooper C, Clarke

$\mathrm{S}$, Fong-Soe-Khioe $\mathrm{R}$, Fordham $\mathrm{R}$, et al. Screening in the community to reduce fractures in older women (SCOOP): a randomised controlled trial. Lancet. 2018;391(10122):741-747.

5. Yang S, Leslie WD, Luo Y, Goertzen AL, Ahmed S, Ward LM, et al. Automated DXAbased finite element analysis for hip fracture risk stratification: a cross-sectional study. Osteoporos Int. 2018;29(1):191-200.

6. Forgetta V, Keller-Baruch J, Forest M, Durand A, Bhatnagar S, Kemp JP, et al. Development of a polygenic risk score to improve screening for fracture risk: A genetic risk prediction study. PLoS Med. 2020;17(7):e1003152.

7. Lee SB, Lee $\mathrm{CH}$, Kim YJ, Kim HM. Biochemical markers of bone turnover in benign paroxysmal positional vertigo. PLoS One. 2017;12(5):e0176011.

8.Høiberg MP, Rubin KH, Hermann AP, Brixen K, Abrahamsen B. Diagnostic devices for osteoporosis in the general population: a systematic review. Bone. 2016;92:58-69. osteoblast differentiation, and inhibiting osteoclasts. Furthermore, it affects adipose tissues, muscle cells, and the neuronal cells that innervate them. It gives a shiny window in the diagnosis, prevention, and treatment of menopause osteoporosis.

\section{Acknowledgements}

This work was supported by College of Health and Medical Technology, Al-Ayen University, Thi-Qar-64001, Iraq.

The authors declare that they have no competing interests.

9.Grygiel-Gorniak B, Puszczewicz M. A review on irisin, a new protagonist that mediates muscle-adipose-bone-neuron connectivity. Eur Rev Med Pharmacol Sci. 2017;21(20):46874693.

10. Al-Badry SH, Khalaf SA, Khalaf, SA AlKhuzaiy AL, Roomi A. The effects of irisin on the transforming growth factor-beta1 (TGF- $\beta 1$ ) level in type-1 diabetic patients in Thi-Qar province. International Journal of Pharmaceutical Research. 2020;12(4).

11. Luo Y, Ma Y, Qiao X, Zeng R, Cheng R, Nie Y, Li S, Shen X, Yang M, Xu CC, Xu L. Irisin ameliorates bone loss in ovariectomized mice. Climacteric. 2020 Apr 21:1-9.

12. Mizil, Oda. (2002). The level of malondialdehyde after activation with (H202 and $\mathrm{CuSO} 4$ ) and inhibition by desferoxamine and molsidomine in the serum of patients with acute myocardial infection. National $\mathrm{J}$ of chemistry. 5. 139-148.

13. Abrahin O, Rodrigues RP, Marçal AC, Alves EA, Figueiredo RC, de Sousa EC. Swimming and cycling do not cause positive effects on bone mineral density: a systematic review. Rev Bras Reumatol Engl Ed. 2016;56(4):345-51.

14. Nadimi H, Djazayery A, Javanbakht M H, Dehpour A, Ghaedi E, Derakhshanian H, et al. The Effect of Vitamin D Supplementation on Serum and Muscle Irisin Levels, and FNDC5 Expression in Diabetic Rats. Rep. of Bio. \& Mol. Bio. 2019;8(3):236-243. 
15. Bischoff-Ferrari HA, Dawson-Hughes B, Orav EJ, Staehelin HB, Meyer OW, Theiler R, et al. Monthly high-dose vitamin D treatment for the prevention of functional decline: a randomized clinical trial. JAMA Intern Med. 2016;176(2):175-83.

16. Roomi AB, AL-Salih RM, Ali SA. The Effect Insulin Therapy and Metformin on Osteoporosis in Diabetic Post-Menopausal Iraqi Women. Indian Journal of Public Health Research \& Development. 2019;10(4):1544.

17. Morris HA, Eastell R, Jorgensen NR, Cavalier E, Vasikaran S, Chubb SA, et al. Clinical usefulness of bone turnover marker concentrations in osteoporosis. Clin Chim Acta. 2017;467:34-41.

18. Piri F, Khosravi A, Moayeri A, Moradipour A, Derakhshan S. The effects of dietary supplements of calcium, vitamin D and estrogen hormone on serum levels of OPG and RANKL cytokines and their relationship with increased bone density in rats. J Clin Diagn Res. 2016;10(9):AF01-AF04.

19. Rowland I, Gibson G, Heinken A, Scott K, Swann J, Thiele I, et al. Gut microbiota functions: metabolism of nutrients and other food components. Eur J Nutr. 2018;57(1):1-24.

20. Deng J, Feng Z, Li Y, Pan T, Li Q, Zhao C. Efficacy and safety of recombinant human parathyroid hormone (1-34) are similar to those of alendronate in the treatment of postmenopausal osteoporosis. Medicine (Baltimore). 2018;97(47):e13341.

21. Chawla H, Saha S, Kandasamy D, Sharma R, Sreenivas V, Goswami R. Vertebral fractures and bone mineral density in patients with idiopathic hypoparathyroidism on long-term follow-up. J Clin Endocrinol Metab. 2017;102(1):251-258.

22. El Wakf AM, Hassan HA, Gharib NS. Osteoprotective effect of soybean and sesame oils in ovariectomized rats via estrogen-like mechanism. Cytotechnology. 2014;66(2):335-43.
23. Kharode YP, Sharp MC, Bodine PV. Utility of the ovariectomized rat as a model for human osteoporosis in drug discovery. Methods Mol Biol. 2008;455:111-24.

24. Manafi Rasi A, ., Amuzadeh Omrani F, Kazemian GH, Ebrahiminia MR, Khani S. Study of Calcaneus Bone Densitometry in PostMenopausal Women. Asian J. Med. Pharm. Res. 2014;4(1):33-4.

25. Kanis JA, Cooper C, Rizzoli R, Reginster JY. European guidance for the diagnosis and management of osteoporosis in postmenopausal women. Osteoporos Int. 2019;30(1):3-44.

26. Colaianni G, Grano M. Role of Irisin on the bone-muscle functional unit. Bonekey Rep. 2015;4:765.

27. Nazirov FG, Khashimov ShKh, Makhmudov UM, Khaybullina ZR, Tuychiev OD. Effectiveness of stage by stage bariatric interventions for regression of comorbidity at obese class III patients. Journal of Life Science and Biomedicine. 2019;9(4):89-95.

28. Zhang J, Valverde P, Zhu X, Murray D, Wu $\mathrm{Y}, \mathrm{Yu} \mathrm{L}$, et al. Exercise-induced irisin in bone and systemic irisin administration reveal new regulatory mechanisms of bone metabolism. Bone research. 2017;5(1):1-4.

29. Palermo A, Strollo R, Maddaloni E, Tuccinardi D, D'Onofrio L, Briganti SI, et al. Irisin is associated with osteoporotic fractures independently of bone mineral density, body composition or daily physical activity. Clin Endocrinol (Oxf). 2015;82(4):615-9.

30. Zhang Y, Li R, Meng Y, Li S, Donelan W, Zhao $\mathrm{Y}$, et al. Irisin stimulates browning of white adipocytes through mitogen-activated protein kinase p38 MAP kinase and ERK MAP kinase signaling. Diabetes. 2014;63(2):514-25. 31. Dun SL, Lyu RM, Chen YH, Chang JK, Luo JJ, Dun NJ. Irisin-immunoreactivity in neural and non-neural cells of the rodent. Neuroscience. 2013;240:155-62. 